01 Электромагнитное излучение при ударном изменении
намагниченности под действием фемтосекундного импульса света

\author{
() В.С. Власов ${ }^{1}$, В.Г. Шавров ${ }^{2, \text { ॠ В.И. Щеглов }}{ }^{2}$ \\ ${ }^{1}$ Сыктывкарский государственный университет, Сыктывкар, Россия \\ ${ }^{2}$ Институт радиотехники и электроники им. В.А. Котельникова РАН, Москва, Россия \\ ๑E-mail: shavrov@cplire.ru
}

Поступило в Редакцию 4 августа 2020г.

В окончательной редакции 8 сентября 2020 г.

Принято к публикации 17 февраля 2021 г.

\begin{abstract}
Выполнена оценка возможности излучения электромагнитной волны при ударном изменении намагниченности под действием мощного импульса света фемтосекундной длительности. В качестве механизма предложена модель ускоренного встречного движения двух разноименных магнитных зарядов. Получены зависимости намагниченности от времени с учетом длительности импульса. Приведены амплитудночастотные спектры излучаемых волн. Определены диаграммы направленности излучения.
\end{abstract}

Ключевые слова: излучение электромагнитной волны, фемтосекундный лазер, магнитный заряд.

DOI: 10.21883/PJTF.2021.11.50997.18498

Возрастающие потребности увеличения быстродействия вычислительных устройств стимулируют освоение процессов, временны́е характеристики которых исчисляются фемтосекундами. Весьма перспективным здесь является исследование сверхбыстрого изменения параметров магнитоупорядоченной среды, вызванного воздействием мощного импульса света от фемтосекундного лазера [1]. Такое изменение обычно реализуется в схеме накачка-зонд, где возмущение магнитных свойств среды, вызванное фемтосекундным лазером (накачкой), регистрируется с помощью зондирующего луча (зонда) от другого лазера с помощью эффекта Фарадея или Керpa [2]. В [3,4] указывается, что за счет мощного импульса накачки малой длительности происходит ударное уменьшение намагниченности среды, вызванное ее локальным нагревом. В цитированных работах отмечено вызванное тепловым ударом возбуждение упругих и магнитостатических волн, распространяющихся только в пределах магнитного образца. В настоящей работе оценивается возможность возбуждения в этих экспериментах электромагнитной волны, выходящей за пределы образца в свободное пространство. В качестве механизма такого излучения предлагается вызванное локальным изменением намагниченности ускоренное движение магнитных зарядов [5-7]. Причиной достаточной эффективности излучения является локальное сверхбыстрое изменение температуры магнитной пленки с соответствующим изменением ее локальной намагниченности. Настоящая работа посвящена рассмотрению возможности такого излучения, а также выявлению некоторых его свойств, в первую очередь спектрального состава и диаграммы направленности.

Будем исходить из общих уравнений электродинамики, включающих в себя кроме электрических также магнитные заряды и токи [8]. Ограничимся рас- смотрением ферромагнетика, структуру которого будем полагать состоящей из магнитных диполей, содержащих два магнитных заряда противоположных знаков. Подчеркнем, что понятие магнитного заряда является вспомогательным и речь идет о том и другом полюсах магнитного диполя. Подобно [5] введем скалярный и векторный магнитные потенциалы, получим для них волновые уравнения и найдем их решения с учетом запаздывания. Для точечных зарядов получаем потенциалы типа Лиенара-Вихерта [9], позволяющие найти поле, создаваемое зарядом, движущимся с ускорением. Проводя выкладки, аналогичные нахождению поля произвольно движущегося электрического заряд [9], для магнитного заряда получаем магнитное поле в виде

$$
\mathbf{H}_{m}=q_{m} \varepsilon_{0} / 4 \pi\left(R-\mathbf{v}_{m} \mathbf{R} / c\right)^{-3}\left[\mathbf{R} \times\left[\left(R-\mathbf{v}_{m} \mathbf{R} / c\right) \times \mathbf{w}_{m}\right]\right],
$$

где $q_{m}-$ магнитный заряд, $\mathbf{R}-$ вектор от точки расположения заряда до точки наблюдения, $\mathbf{v}_{m}$ и $\mathbf{w}_{m}$ скорость и ускорение движения заряда, $c$ - скорость света.

Полагая $v_{m} \ll c$, а также представляя магнитный заряд как произведение поверхностной плотности заряда $\mu_{0} M$, где $M$ - намагниченность насыщения, на площадку $S$ и учитывая, что магнитные заряды всегда присутствуют в виде диполя, так что поля от их встречного движения складываются, приводим (1) к виду

$$
\mathbf{H}_{m}=2 M S \mu_{0} \varepsilon_{0} R^{-3}(4 \pi)^{-1}\left[\mathbf{R} \times\left[\mathbf{R} \times \mathbf{w}_{m}\right]\right] .
$$

Полученное поле по абсолютной величине обратно пропорционально первой степени расстояния от заряда до точки наблюдения $R$, что указывает на распространение электромагнитной волны.

Положим теперь, что скорость движения заряда пропорциональна скорости изменения намагниченности; то 
же касается ускорения. Изменение намагниченности под действием мощного импульса света во времени состоит из двух частей: в течение длительности импульса температура магнитной среды повышается, так что намагниченность уменьшается, а после его окончания температура падает, так что намагниченность возрастает $[3,4]$. Типичная зависимость намагниченности от времени при различных длительностях импульса показана на рис. 1. В качестве опорных были взяты параметры пленки никеля [3,4], имеющего намагниченность насыщения $4 \cdot 10^{4} \mathrm{~A} \cdot \mathrm{m}^{-1}$ и температуру Кюри около $630 \mathrm{~K}$. Выделяемая импульсом энергия такова, что перепад температур, например, для кривой 5, составляет от 300 до $590 \mathrm{~K}$, что приближается к температуре Кюри. В продолжение импульса намагниченность падает, а после его окончания растет, стремясь к первоначальному значению. Крутизна кривых, определяющая ускорение магнитного заряда, до окончания импульса с увеличением его длительности убывает, а после его окончания возрастает, т.е. для коротких импульсов основное излучение имеет место при нагревании пленки, а для длинных - при охлаждении.

На рис. 2 показаны спектры излучаемых электромагнитных волн при тех же длительностях импульса, что и на рис. 1. Наиболее широкий спектр (кривая 1) имеет место при наименьшей длительности импульса. По мере увеличения длительности спектр сужается, а начиная с 0.40 ps (кривая 4) при дальнейшем увеличении (кривая 5) не меняется.

Ввиду геометрических свойств векторного произведения в формулах (1), (2) следует ожидать, что распределение излучения в пространстве будет резко анизотропно. Рассмотрим такое распределение. В [6] показано, что диаграмма направленности излучения, создаваемого ускоренным движением одного магнитного заряда, с

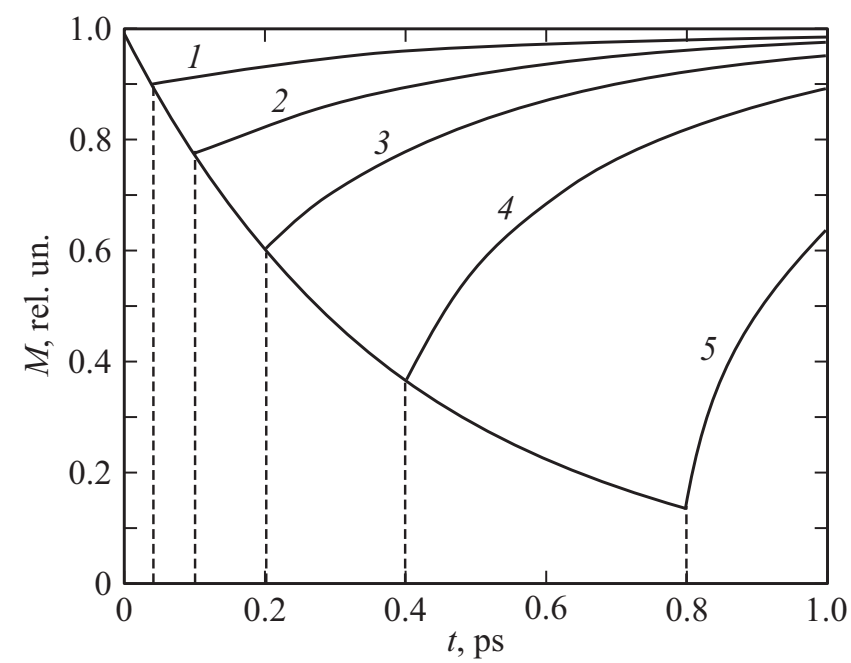

Рис. 1. Зависимость намагниченности от времени при различных длительностях импульса (ps): $1-0.05,2-0.10,3-$ $0.20,4-0.40,5-0.80$. Намагниченность нормирована на значение при температуре $300 \mathrm{~K}$.

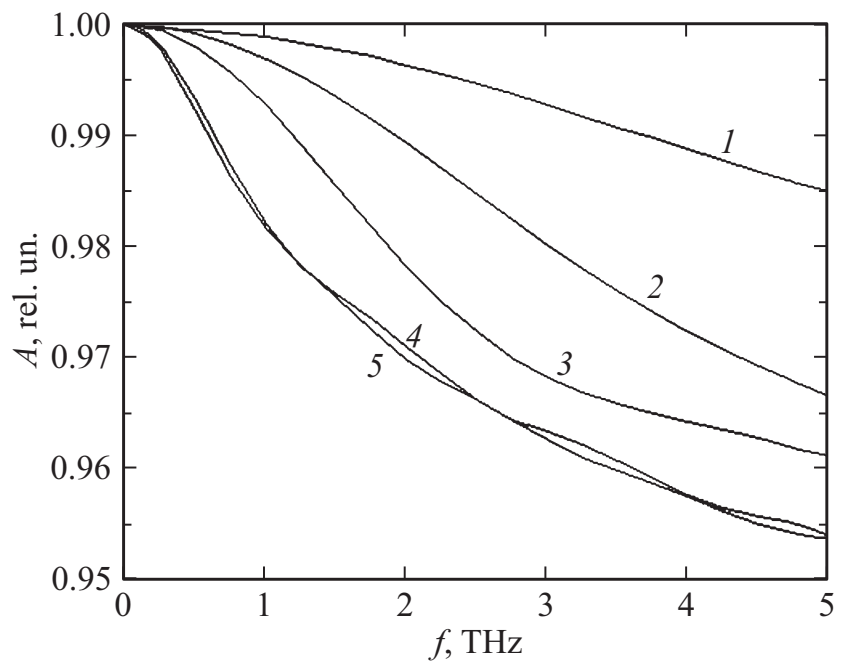

Рис. 2. Спектры излучаемых электромагнитных волн при различных длительностях импульса (ps): $1-0.05,2-$ $0.10,3-0.20,4-0.40,5-0.80$. Максимальная частота соответствует $5 \mathrm{THz}$. Амплитуды нормированы на значение при нуле.

точностью до нормирующего множителя $A$ имеет вид

$$
U=A w_{m}^{2}\left[\sin \alpha\left(1-v_{m} c^{-1} \cos \alpha\right)^{-2}\right]^{2}
$$

где $U-$ плотность энергии излучаемой волны, $\alpha-$ угол между осью движения заряда и направлением на точку наблюдения, причем коэффициент $A$ в соответствии с (2) обратно пропорционален второй степени расстояния от заряда до точки наблюдения.

На рис. 3 показаны диаграммы направленности излучения при различных длительностях импульса. При этом значение $A$ принято единым и нормировано на максимум. Движение заряда, его скорость и ускорение направлены вдоль оси $O x$, перпендикулярной плоскости пленки, ось $O y$ лежит в плоскости пленки. Из рисунка видно, что диаграммы при любой длительности импульса по ориентации геометрически подобны друг другу. При этом в условиях сохранения энергии импульса (т. е. величины коэффициента $A$ ) чем импульс короче, тем излучение сильнее, что обусловлено большей величиной ускорения движения заряда для более коротких импульсов.

Выполним численную оценку направленности и интенсивности излучения для параметров, типичных в экспериментах по схеме накачка-зонд [1-4]. Пусть пленка имеет форму квадрата со сторонами по $100 \mu \mathrm{m}$ и обладает толщиной $10 \mathrm{~nm}$. Ускоренное движение зарядов происходит по толщине пленки. Предполагаем, что за время действия импульса заряд проходит путь от одной поверхности пленки до другой с постоянным ускорением. При длительности импульса $0.2 \mathrm{ps}$ ускорение получается равным $5 \cdot 10^{17} \mathrm{~m} \cdot \mathrm{s}^{-2}$, при длительности импульса $0.02 \mathrm{ps}-5 \cdot 10^{19} \mathrm{~m} \cdot \mathrm{s}^{-2}$, при длительности $0.40 \mathrm{ps}-1.2 \cdot 10^{17} \mathrm{~m} \cdot \mathrm{s}^{-2}$. Максимальная скорость во 


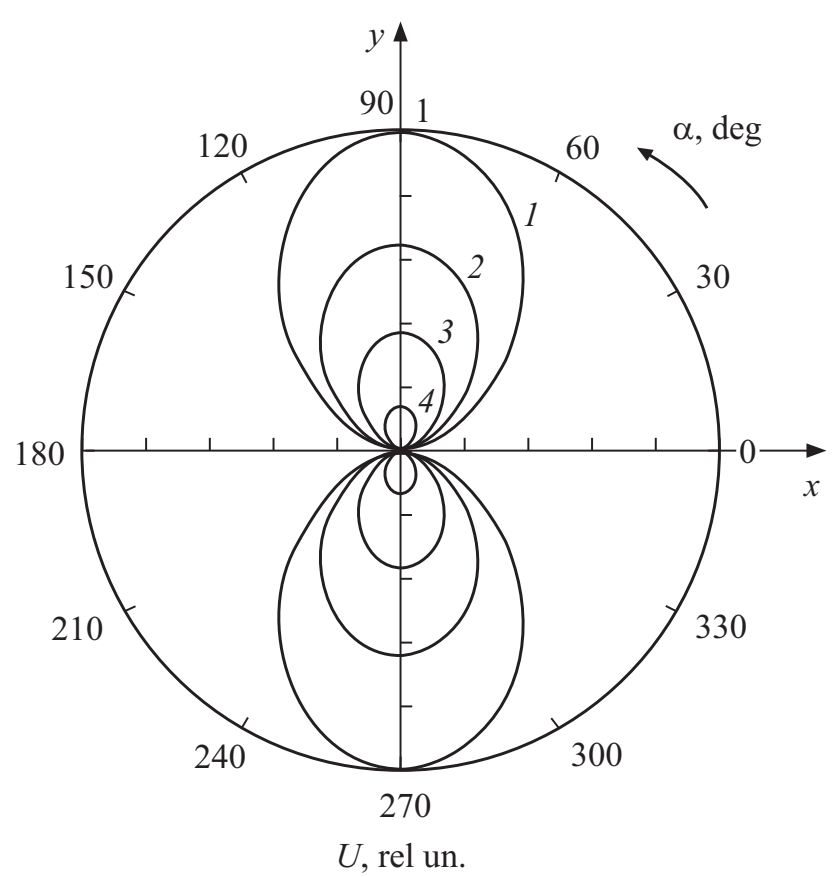

Рис. 3. Диаграммы направленности излучения при различных длительностях импульса $(\mathrm{ps}): 1-0.10,2-0.12,3-0.15$, $4-0.20$. Амплитуды нормированы на значение при длительности $0.10 \mathrm{ps}$.

всех этих случаях получается не больше $10^{6} \mathrm{~cm} \cdot \mathrm{s}^{-1}$, что значительно меньше скорости света, поэтому роль второго слагаемого в круглых скобках в формуле (3) незначительна, так что все диаграммы на рис. 3 практически симметричны относительно оси $O y$. Теперь с помощью формулы (3) оценим поле волны на некотором расстоянии от пленки никеля, имеющей намагниченность порядка $4 \cdot 10^{4} \mathrm{~A} \cdot \mathrm{m}^{-1}$. Поскольку излучение рассматривается в дальней зоне $[8,9]$, т.е. на расстоянии, значительно большем размеров образца $(100 \mu \mathrm{m})$, будем отсчитывать расстояние в $1 \mathrm{~cm}$ от центра пленки. При длительности импульса $0.2 \mathrm{ps}$ поле в точке наблюдения равно $3 \cdot 10^{-2} \mathrm{~A} \cdot \mathrm{m}^{-1}$, при длительности импульса $0.02 \mathrm{ps}-$ $3 \mathrm{~A} \cdot \mathrm{m}^{-1}$, при длительности $0.40 \mathrm{ps}-7.5 \cdot 10^{-3} \mathrm{~A} \cdot \mathrm{m}^{-1}$. Чем короче импульс, тем поле больше, что также видно из рис. 3. Следует отметить, что поля такой величины можно легко наблюдать в эксперименте. Регистрацию излучаемой электромагнитной волны можно выполнить с помощью стандартного измерительного приемника с рупорной антенной такого типа, как в экспериментах, описанных в работах [5-7].

Таким образом, главным результатом работы можно считать количественную оценку интенсивности излучения электромагнитной волны при ударном изменении намагниченности под действием мощного импульса света фемтосекундной длительности, выполненную на основе модели ускоренного встречного движения двух разноименных магнитных зарядов.

\section{Финансирование работы}

Работа выполнена в рамках государственного задания Института радиотехники и электроники им. В.А. Котельникова РАН.

\section{Конфлликт интересов}

Авторы заявляют, что у них нет конфликта интересов.

\section{Список литературы}

[1] J. Walowski, M. Münzenberg, J. Appl. Phys., 120 (14) 140901 (2016). DOI: 10.1063/1.4958846

[2] A. Kirilyuk, A.V. Kimel, T. Rasing, Rev. Mod. Phys., 82 (3), 2731 (2010). DOI: 10.1103/RevModPhys.82.2731

[3] E. Beaurepaire, J.C. Merle, A. Daunois, J.Y. Bigot, Phys. Rev. Lett., 76 (22), 4250 (1996).

[4] J.V. Bigot, M. Vomir, Ann. Phys. (Berlin), 525 (1-2), 2 (2013).

[5] В.Г. Шавров, В.И. Щеглов, Магнитостатические и электромагнитные волны в сложных структурах (Физматлит, М., 2017).

[6] В.И. Зубков, В.И. Щеглов, Радиотехника и электроника, 46 (4), 433 (2001).

[7] В.И. Зубков, В.И. Щеглов, Письма в ЖТФ, 34 (22), 44 (2008).

[8] А.А. Семенов, Теория электромагнитных волн (Изд-во МГУ, М., 1968).

[9] В.Г. Левич, Курс теоретической физики (Наука, М., 1969), т. 1. 